SÁND-79-1129

UNLIMITED DISTRIBUTION

COMPUTATIONS OF THE SPHERICAL E $\rightarrow 1$ CONVERTOR

J. W. POUKEY 
SAND-79-1129

\author{
Computations of the Spherical e $\rightarrow$ i convertor \\ James W. Poukey
}

\begin{abstract}
One proposed approach to the overlap/deposition problem for multi-channel electron beams is to place a thin hollni sphere in the overlap region and allow the incoming electron: to form a victual cathode inside this sphere. The resulting flectric field causes ions from the inner surface of the sphere to Hiow inward $r=\rightarrow i a l l y$ towards a target. The efficiency of this process is studied analytically and with a 1-D spherical, two-species electrostatic particle code. The maximum possible (powel conversion) efficiency is concluded to te about 10 percent. Two-dimensional effects are briefly considered, and angul-. momentum in particular is found to be a possitly serious limitation.
\end{abstract}




\section{TABLE OF COITENTS}

Page

I. Tr.e Easic coheme. . . . . . . . . . . . . . 5

¿. analyt $\because$.

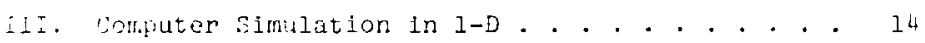

I. Esulble 2-D Effocts. . . . . . . . . . . . 18

y, conclustons. . . . . . . . . . . . . . . 20

r.o.rerces. . . . . . . . . . . . . . . . . 22

Mtble I - Sumary of 1-D Simulations. . . . . . . 16

Firuro 1 - Echematic of e - i Convertor....... 6

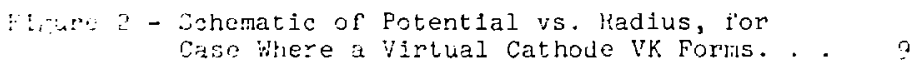




\section{THE BASIC SCHEME}

There are a number of questions cuncerning direct use or the electrons in a REB multi-channel scheme. These concern yetting the electrons to a small (am-sized) target in the overlap region, and the efficiency of deposition in the target if the eleztrons do get there.1,2,3 one froposed way to avoid these problems is to convert the e-beams to $i$-beams using a hollow sphere of radius $r_{l}($. few $\mathrm{cm}$ ) as indicated in Fig. 1.4 Ideally, nost olectrons will make several passes through the sphere at ' ${ }^{\prime}$ ' being reflected on the inside by a virtual cathode $V k$ and on the putside by the magnetic field of the combined channel curcents. An electron's ultimate fate is either l) absorption in the shel: at $r_{1}$, 2) drifting away outside of $r_{1}, 0:$ 3) loss to the taryet at $r_{2}$. A plasma will form on the inside of the shell at $r_{l}$, and space-charge-limited ions will fiow inward Lowards the target.

This type of fusion scheme has a long nistury. It is basicaily the same as suggested by Goldstein and Guillory, j except that here the shell is placed in the overlap region of the REB-channel system instead JF inside a single djode. Similar ideas have bien advanced by $V: r$ deyen ${ }^{5}$ and earlier by Hirsch, ? although at lower current livels. Computel calculations of the 1-D spherical inertia? corfinement device were performed by Barnes: ${ }^{8}$ The work reported in the present paper differs from this last reference in (1) injecting electrons, and allowing space-charge-limsted ions, (2) including relativistic (but not magn tic) efferts, and (3) considering much higher current 


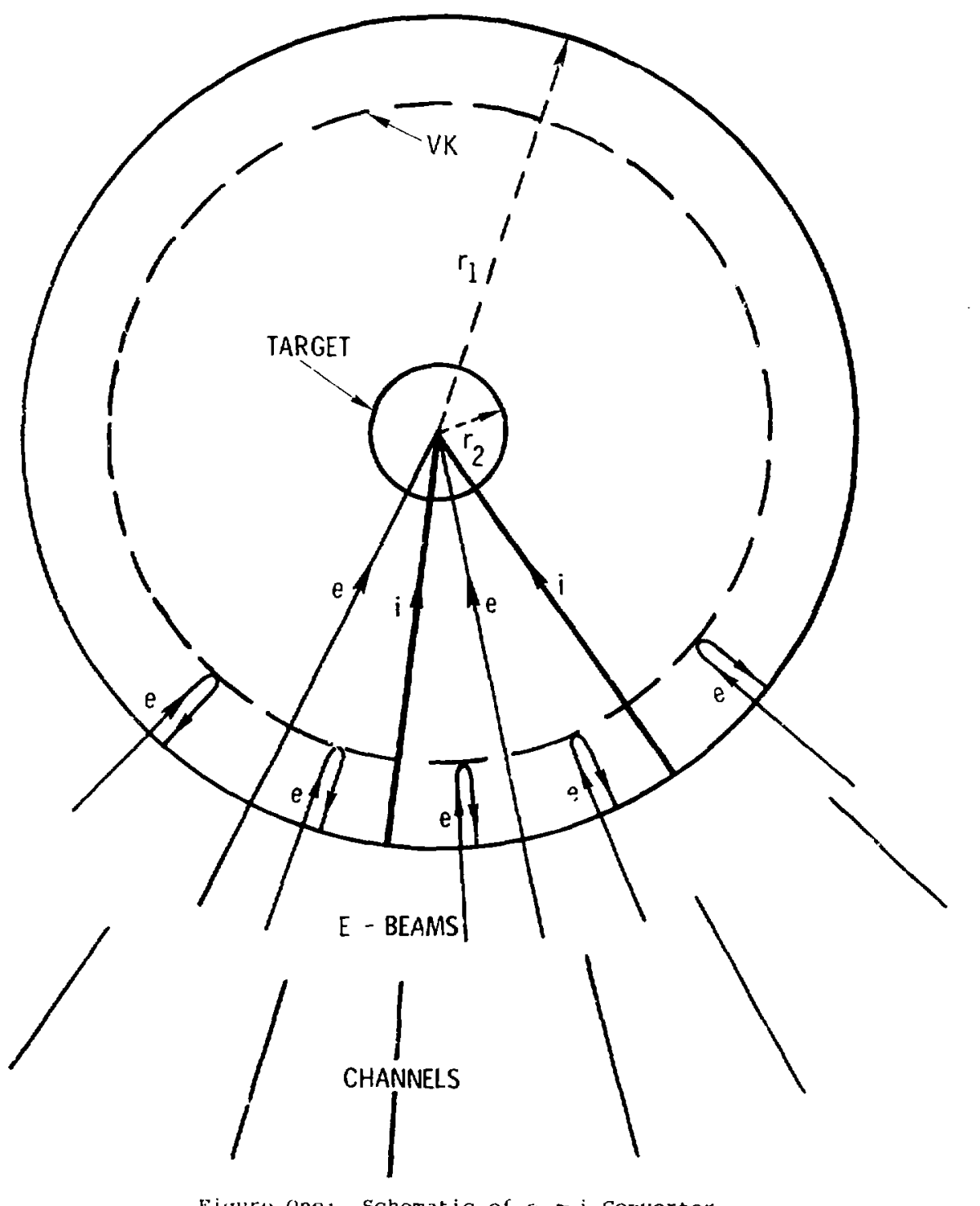

Figure one: Schematic of $c \rightarrow$ i Convertor

5 
dansities. Nevertheless, several features observed in the present work were aisu seen by Barnes, as will be pointed mit later.

The main question concerniny the scheme is the efficiency with which electron energy can be converted to ion energy incident on the target. The calculations will be time-independent in the sense that only equilibria, or "quasi-nquilibria" (where the system oscillates about a defined average state), will be computed. Thus we shall not consider obvious questions such as electron pes-heat, plusma formation or motion, or pulse shaping or other methods to obtain ion bunching. Hence the "efflciency" will be deternined simply by $I_{i} \ddot{E}_{i} / I_{e} E_{e}$, where $I_{i}$ is ion current to the tariget, $\bar{E}_{i}$ is average ion energy to the target, and $I_{e}$ and $E_{e}$ are the curcent and energy of the incisent elcctrons.

The computations to be discussed are one-dimensional in the siherical coorilinate $r$. This was done despite the fact that the te ar? a number of obviously important 2-D (and 3-D) effects in any real system, such as magnetic fields, angular momentun, asymmetry in the incoming e-beam, return curreni path, nonuniformity in the ion-enitting plasma, etc. The first reason for adoptir, a 1-D model for this problem is purely practical. Fot the current levels of interest, the appronciate length scales walih must be resolved are of order $c /-b \cdots<r_{1}$. This makes even $1-D$ code calculations relatively expensive. Attumpts were made to model the problem in 2-D, nevertheless, using a previously developed spherical diode code which included ragnetic effects, 9 but all such efforts thus far have falled 
to produce useful solutions. A second complicating feature of the present problem is that unlike an ordinary spherical diode, which has been studied analytically, 10,11 the inner sphere at $r_{2}$ is floating in potential so that we have vanishing electric field boundary conditions at both $r_{l}$ and $r_{2}$. Physically this means that the total electron and ion fluxes to $r_{2}$ must be equal, and that the net charge in the system $r<r_{l}$ must be zero. This is an easy condition to enforce in $1-D$, but is much more difficult in 2-D. A third reasin for using a $1-0$ model is that all the 2-D

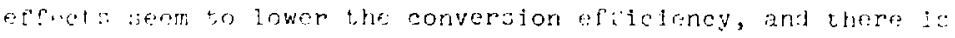

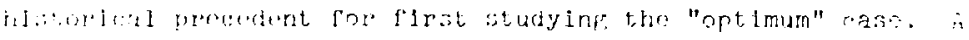

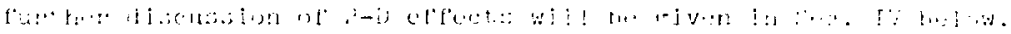

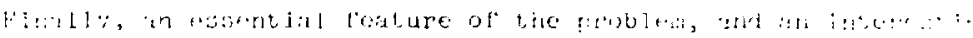

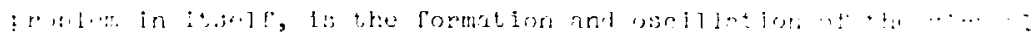

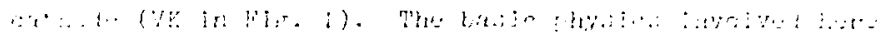

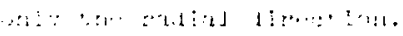

II. ANALYTIC MODEL

The potential $g(r)$ in the expocted solution is sketched in Fig. 2. The electrons enter the conducting sphere at $r_{1}$ with some ladial velocity ${ }^{\prime}$, and may form a virtual cathode at Vk, where $\emptyset=\emptyset_{\mathrm{min}}$. Some electrons turn arounj at $\mathrm{VK}$ and go back to ${ }_{1}$. while some continue on to hit the target at $r_{2}$. Space-charge-limited ions flow inward $f r a m r_{1}$ to $r_{2}$, and the ion current $r_{i}$ must equal the electron current $I_{e}$ to the target. Except for a reversal of species, this is just the "type two" steady state of Barnes. 8 


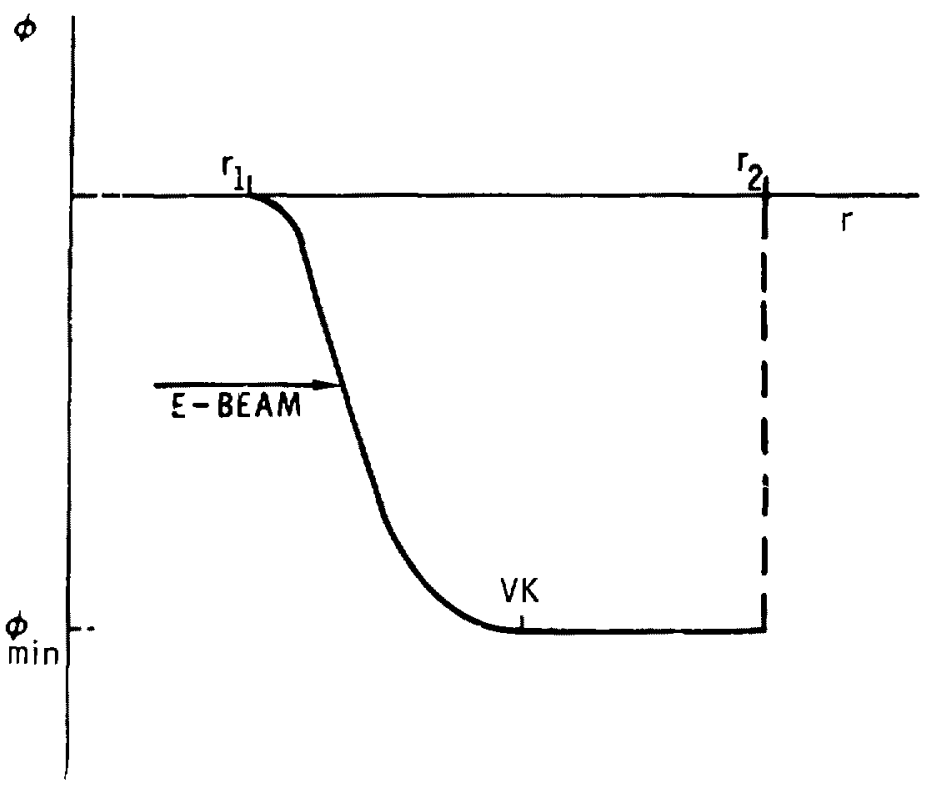

Fichte Two: Schematic of Potential vs. Radius, for Case Where a virtual cathode vi Forms

9 
If the injected e-beam is cr.ld (nu spread in $v_{0}$ ), then in the static solution $\oiint_{\text {in in }}$ must equal the injected enorgy ev, where $V$ corresponds to the voltage of the diode producing the beam ineglecting losses of energy in the channel and the sphere). Such a system is intrinsically unstable, 12, i3 because an infinitesimal perturbation in $\varnothing_{m i n}$ results in a finite change in the curcent $I_{e}$ (either all or nothing). A system into which a truly cold beam is injected will avoid this problem by not forming a steady state; as shown previously, 14 such a system will oscillate furever, with $\emptyset_{\text {inin }}$ someuimes above, sometines below, $V$. This will alluw (on the average) the proper $\mathrm{I}_{\mathrm{e}}=\mathrm{I}_{\mathrm{i}}$ to be drawn by the taryet.

Any real system, however, will have some spread in $v_{0}$, and according to provious work, 8 although a static 5, tution is still unstable in the sense of possessing oscillations, these oscillations may be controlled tc some extent by increasing the initial velocit: spread. In choosing a model for the e. I convertor, we must recall that the injected electrous will be experiencing complicated magnetic and 2-D electric fields, foil scittering and enerjy loss, and will possess considerable angular nomentum. In terms of a 1-D model, in fact, the e-beam will be very "hot", so for the incoming $r a d i a l$ velocity distribution we will simply say

$$
\mathrm{f}_{\text {in }}\left(\mathrm{r}_{1}, \mathrm{v}_{0}\right)= \begin{cases}\mathrm{f}_{0} & 0 \times v_{0}<v_{d} \\ 0 & v_{0}>v_{3}\end{cases}
$$


where $s_{0}$ is a constant, and $v_{d}=c\left(1-1 /\left(1+e v / m^{2}\right)^{2}\right)^{1 / 2}$ is the maximum possible radial velocity.

The total injected electron current is found to we

$$
I_{\text {in }}=N I_{\text {exp }}=2 \operatorname{Ier}{ }_{1}^{2} E_{o} c^{2}\left(1-1 / \gamma_{d}^{2}\right)
$$

where $r_{d}=\left(1-v_{d}^{2} / c^{2}\right)^{-1 / 2}, I_{e x n}$ is the actual current

flowing down the channels, and $N$ is the average number $c t$ passes through the foil at $r_{1}$. The electror current hitting the target, and hence the ion current, is then

$$
I_{i}=2 \pi \operatorname{er}_{1}^{2} f c_{o}^{2}\left(\frac{1}{\left(1-\eta_{m i n}\right)^{2}}-\frac{1}{r_{d}^{2}}\right)
$$

where $\eta_{\text {min }} \equiv e \emptyset_{m i n} / m^{2}$, m beins the electron mass.

Therefore,

$$
I_{i}=i_{i n}\left(\frac{r^{2}}{\left..1-n_{\min }\right)^{2}}-1\right) \frac{I}{r_{a}^{2}-1}
$$

ana since $r_{d}$ is known, it remains to $E$ ind "min. (Note tre obvious resu $t$ that if $\left.g_{\min }=-v, I_{i}=0.\right)$

We follow the time-honored procedure of rinciag the electron and ion densities $n$ terms of $g$, or $n=e \emptyset / \mathrm{mc}^{2}$, and obtain the following boundary-value problem: 


$$
\begin{aligned}
& \frac{d}{d r} r^{2} \frac{d \eta}{d r}=\frac{I_{e}}{8.5 k A}\left[2 \int_{I-n}^{\gamma_{m}} \frac{d o}{r_{0}^{3}\left(1-1 /\left(\gamma_{0}+n\right)^{2}\right) 1 / 2}\right. \\
& +\int_{r_{m}}^{r_{d}} \frac{d r_{0}}{r_{0}^{3}\left(1-1 /\left(r_{0}+n\right)^{2}\right)^{1 / 2}} \\
& -\frac{1-\frac{r_{m}^{2}}{2}}{2\left(\frac{2 m}{m_{i}}\right)^{1 / 2} r_{d}^{2}(-n)} \frac{\frac{r_{c}^{2}}{r_{d}^{2}-1}}{r^{2}}, \\
& \eta^{\prime}\left(r_{1}\right)=\eta \cdot\left(r_{2}\right)=n\left(r_{1}\right)=0
\end{aligned}
$$

where prime means $d / d r$ and $\gamma_{m} \equiv 1-\eta_{m i n}$ is the smallest value of $y$ which can reach the target. Here $0 \approx\left(1-v_{0}{ }^{2 / c^{2}}\right)^{-1 / 2}$. we note that in this model, there is no well-defined virtual cathode, since electrons are turning around at all radii. The expected solution for $\varphi(r)$ is still basically like that in Fig. 2 , except that $f(r)$ will decrease all the way in to the target $\left(r_{2}\right)$. Equation (5) is a second order differential ec:ation for $n(5)$, and the extra boundary condition (6) can be used to find $n_{\min }=1-Y_{m}$, at iesst in principle. In practice, Eq. (5) does not seem to have an analytic first integral, so we resoct to making a rough approximation. Ignoring spherical effects by assuming $(d / d r)\left(r^{2} d n / d r\right)=r^{2} d^{2} n / d r^{2}$ for some $r *$ in $\left(r_{2}, r_{1}\right)$, we get $\frac{1}{2}(d n / d r)^{2}=r^{-2}, \rho(n) d n$ where $\rho$ j.s the right side of Eq. (5). Using Eqs. (6) leads to 


$$
\begin{aligned}
& 2 \int_{1}^{i m} \frac{d y}{r^{3}}\left(r^{2}-1\right)^{1 / 2}+\int_{r_{30}}^{y d} \frac{d y}{r^{3}}\left(\left(r^{2}-1\right)^{1 / 2}\right. \\
& \left.-\left|(\gamma+n \min )^{2}-1\right| 1 / 2\right) \\
& =\frac{\left(-n_{\min }\right)^{1 / 2}\left(r_{\mathrm{m}}^{\frac{1}{2}}-\frac{1}{r^{2}}\right)}{\left(2 \mathrm{~m} / \mathrm{m}_{i}\right)^{1 / 2}} .
\end{aligned}
$$

As a crude approximation, if $Y_{G} \cdot Y_{m} \cdots 1$,

$$
\cos ^{-1} \frac{1}{y_{m}} \quad-{ }_{2}^{1} \sin \left(2 \cos ^{-1} \frac{1_{-}}{\gamma_{m}}\right)=\sqrt{\frac{m_{i}}{2 ! n}}\left(\frac{1}{\gamma_{m}^{2}}-\frac{1}{\gamma_{d}^{2}}\right) \sqrt{-n_{m i n}}
$$

or roughly,

$$
\left(r_{m}-1\right)\left(\frac{1}{r_{m}^{4}}-\frac{2}{r_{j}^{2} r_{m}^{2}}+\frac{1}{r_{d}^{4}}\right)=\left(\begin{array}{l}
\pi \\
2
\end{array}\right)^{2} \frac{2 m}{m_{i}}
$$

Dr

$$
Y_{m}=Y_{d}-\frac{\pi}{2} \sqrt{\frac{m}{2 m_{i}}} \gamma_{d}^{5 / 2},
$$

so that we find from Eq. (4)

$$
I_{i}=I_{e_{i r_{i}}} \pi \sqrt{\frac{m}{2 m_{i}}} \frac{1}{\sqrt{y_{d}}}
$$

According to this result, the conversion from electron to ion current will be rather inefficient (a few 8 ), unless $t_{e}=N_{e}$ 
can be insedica substantially by forcing the averuge number of passes $N$ tor be al lnast $\sim 10$.

\section{Computer Simulation in, $!-D$}

s una-danemioral particle simulation cos: wat writton

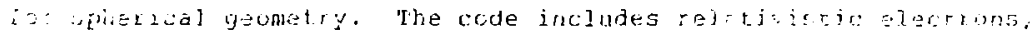

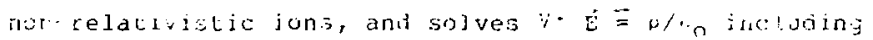

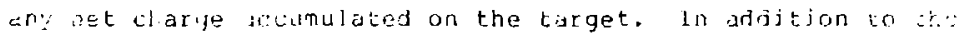

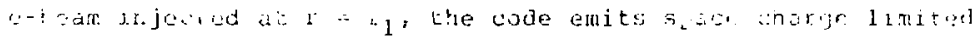

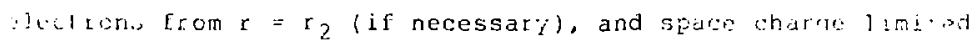

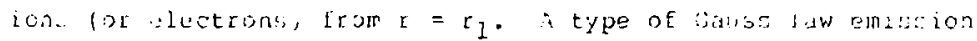

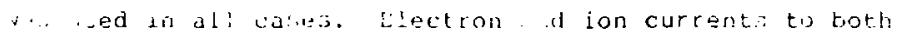

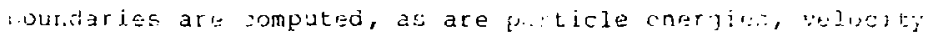
djas ibutans, etc.

The lirst cases studied (as tests of the coiel wore those 1. whith ontr the injerrat electron beam was inotudert it: : a woli-derined VK lorins at about $r=r_{1}-c / . h$, whern $\omega_{b}=$ beam

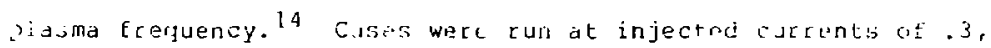
3, and $30 \mathrm{MA}$ with cold beams at $2 \mathrm{MeV} ; \mathrm{c}_{1}=4.8 \mathrm{~cm}$. No electrons reacin the target ac these curcents. Besides testing the code, these runs provided inforliation on the requires zoning, which of necessity becomes very fine at the higher injected currents, because of the need to adequately resolve $c / \omega_{b}$, which is about $\operatorname{lm} \pi$ at 30MA. This zoning requirement at currents of interest in turn inade it necessary to use more particles $\left(\sim 10^{4}\right)$, and made the runs relatively expensive for a l-D simulation. 
The early runs including ions were done at an artificial mass ratio, $m_{i} / m=100$, to more efficiently studv the oscillations which were present in all runs, and the deperiugence of the solutions on time step, particle number, cell size, and enission method, All remaining runs (discussed below) were done with $m_{i}=$ proton mass. The code confirmed the $I_{i}=m_{i}^{-1 / 2}$ scajing cf Eq. (8) .

Table I gives a summary of the main simulations. Runs $1-8 \mathrm{had} \mathrm{r}_{1}=3 \mathrm{~cm}, \mathrm{r}_{2}=1 \mathrm{~cm}$, and wore for parameters possibly relevant to EBFA. ${ }^{4}$ Runs $9-11$ had $r_{1}=4.4 \mathrm{~cm}, r_{2}=1.1 \mathrm{~cm}$, and were intended to be relevant to the upcoming Hydra experiments of P. Miller.15 The injected 8.8 MA in the latter comes from a large assumed source radius of $1.5 \mathrm{~cm}$, with . $25 \mathrm{MA}$ per channel: $\mathrm{I}_{\mathrm{e}} \doteq .25\left(4 \pi \mathrm{r}_{1}^{2} / \pi(1.5 \mathrm{~cm})^{2}\right)$. In the table, $E_{e}$ is the injected e-beam orergy, $T_{e}$ describes the beam as cald (no spread in injected radial velocities) or hot (velocities selected randomly from $\left.\left(0, v_{d}\right)\right)$. The last four columns in the table describe the results: $I_{i}$ and $\bar{E}_{i}$ are the ion current and average energy to the target at $r_{2}$, VK is the position of the virtual cathode (if one forms), and P. E. is the power efficiency, defined as $\bar{E}_{i} I_{i} / E_{e} I_{i n}$ with $E_{e}$ ceplaced by $\bar{E}_{e}$ in cases where a rot beam is injected.

In run \#1, $30 \mathrm{MA}$ of electrons, ail at $2 \mathrm{MeV}$, is converted to about 2.6 MA of $2.6 \mathrm{M} \geqslant \mathrm{V}$ protons. The VR forms close to $\mathrm{r}_{1}$ because the current is so large. In runs 2-5, lower energy beams (each cold) were added to the 30MA, to simulate the 
Table I. Summary of l-s Simuiations

\begin{tabular}{|c|c|c|c|c|c|c|c|c|c|}
\hline Run & $r_{1}(\mathrm{~cm})$ & $\tau_{2}(\mathrm{~cm})$ & $\mathrm{E}_{\mathrm{e}}(\mathrm{MeV})$ & $I_{\mathrm{in}}(\mathrm{MA})$ & $T_{12}$ & $I_{i}(M A)$ & $E_{i}(\mathrm{MeV})$ & $v K(\mathrm{~cm})$ & P.E. \\
\hline 1 & 3 & 1 & 2 & 30 & $\operatorname{col} d$ & 2.55 & 2.64 & 2.84 & 118 \\
\hline 3 & 3 & 1 & $\left(\begin{array}{r}2 \\
.31 \\
.12\end{array}\right.$ & $\begin{array}{l}30 \\
15 \\
15\end{array}$ & $\begin{array}{l}\text { cold } \\
\text { cold } \\
\text { cold }\end{array}$ & 3.0 & 2.6 & 2.84 & 128 \\
\hline 4 & 3 & 1 & $\left(\begin{array}{l}2 \\
.31 \\
.12 \\
.04 \\
.01\end{array}\right.$ & $\begin{array}{l}30 \\
10 \\
10 \\
10 \\
10\end{array}$ & $\begin{array}{l}\text { cold } \\
\text { cold } \\
\text { cold } \\
\text { cold } \\
\text { cold }\end{array}$ & 2.8 & 2.5 & 2.84 & 118 \\
\hline 7 & 3 & 1 & $\left(\begin{array}{l}2 \\
0-2\end{array}\right.$ & $\begin{array}{r}6 \\
24\end{array}$ & $\begin{array}{l}\text { cold } \\
\text { hot }\end{array}$ & .7 & 2.5 & --- & $1 C ?$ \\
\hline 8 & 3 & 1 & $0-2, \overline{\mathrm{E}}_{\mathrm{e}}=. ?$ & 30 & hot & .6 & 1.9 & --- & 198 \\
\hline 9 & 4.4 & 1.1 & 1 & 8.8 & $\operatorname{cold}$ & .3 & 1.9 & 4.2 & 178 \\
\hline 10 & 4.4 & 1.1 & $0-1, E_{\mathrm{e}}=.18$ & 8.8 & not & .2 & 1.1 & --- & 148 \\
\hline 11 & 4.4 & 1.1 & $0-1, \ddot{E}_{e}=.18$ & 35 & not & .9 & 1.5 & -- & 218 \\
\hline
\end{tabular}


situation where the electrons make several passes through the foil but with mucil reduced energy each pass. It is seen from the table that ajding these extra beans had no substantial effect on the conversion to ions; the cold 30MA beam completely dominates. In runs 6-8, we go from the limit of perfectly cold to extremely hot, keeping the total $I_{\text {in }}$ constant. In fact run 8 is the supposedly realistic case which we adopted for our analytic model in Ses. II, wherein we assumed the radial velocities were uniformly distriblted over the interval $\left(0, v_{d}\right)$. In runs $i, 6,7,8$ the trend is for the virtual cathode to move in towards the target and vanish, after which the $\mid \theta_{\text {min }}$ " $v$, where Min sccurs at the taryet. There is also a trend of decreasinu ion current; the value predicted by Eq. (8) is $.7 \mathrm{MA}$, in surprisingly grod agreement with the code ( $\mathrm{r}$ un 8 ; considering the rough approximations made in deriving Eq. (8). Next we discuss the bydra runs 9-11. The cold-beam rin 9 is similar to the EBFA cun I, having slightly higher PE presumably tuacause of the lower $\mathrm{d}^{\mathrm{S}}$. Run 10 just injects the $8.8 \mathrm{MA}$ as a not beam, assuming each electron makes only one pass into the system $(N=1)$, whereas $x$ un 11 assumes $N=4$, considered by some to be the optimum in ordinary reflex diodes. 16 Neicher 10 nor 11 formed a VK. The predictions of Eq. (8) for these cases are .26 and $1.05 \mathrm{MA}$, respectively, and we see from the table that the agreement between the code and the analytic model is good enough to warcant taking $\mathrm{Eq}$. (8) with some seriousness. The PE values in runs 8,10 , and 11 , however, are not to be taken 
too seriously, isecause in dividing ty $E_{e}$, the average ratial enfigy, they overestimate the e[ficiency by new]ecting thr. ron-radial kinetic energy. Uaing $F_{e}=1$ Mev instad, the PE valusis in 10 and 11 becume 33 and $4 \%$. On the other hand, in rum 1 l one should resnmber that $\mathrm{N}=4$ so that the "realistic" Pr: here is 15\%. As a semi-empirical result, we may write approximately

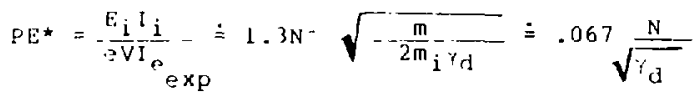

fir the hot bean cases. To pursue the efficiency question turther here would be to stretch the applicability of the :-l) model beyond reasonable limits, but it is interesting that most of the PE values we have obtained are about 10 to 158 ; to qdot: Fef. 16 on the behavior of 1-D reflex diodes, ... in o. sinulations, the ion current efficiency was about 508 . Nevirtheloss, when remembering that we used $m_{i} / m_{e}=25$, the result for a real in $/ m_{e}$ ratio would be correspondingly lower, in agreement with the maximun theoretical estimate o $10-15 \mathrm{~s} . "$

\section{Possible 2-D Effects}

The two or three dimensional problem, including magnetic fields, angular momentum, etc., seems to be quite intractable analytically. And, for reasons which should now be apparent, a straightforward 2-D code is not feasible either, although this does not preclude the $p^{\prime}$ isibility of making some simplifying assumptions concerning the electron dynamics, and/or using 
ron-uniform grid spacing to help with the severe resolution requirements For present purposes, we inention what may $m$ the nost serious 2- or 3-D effects, all of wion tend to make our $1-[$ results er on the optimistic side.

The first problem is that there will be serious spherical asymetries in the electron behavior, and conszguently in the ion trajectories. The incoming e-beams will intercept the spherz at $r_{1}$ with a built-in asymmetry, and the magnetic fields near $r_{l}$ will have a complicated dependence on all three coordinates. Inside $r_{1}$ there will be non-radial electric fields which will prevent the ions from focusing at the center. Despice resolution and boundary andition difficulties, a 2-D $(\mathbf{r}, 1)$ particle code was attempted, based on a previously dnveloped spherical diode code. Although no acceptable steaty solutions were ever found with this code, the work clearly showed the asymmetries in fields and ion trajoctories.

The second problem, as pointed out by $P$. Miller, 4 is the loss of electrons outside $r_{1}$, due to the vB (and possibly otter) drifis. The preliminary work with the $2-D$ code suggested this is not as severe a problem as might be expected, although no definitive statement can yet be maje. Of course, this effect has not been included in the $1-0$ model of the present paper, but it would show up in the equations as a decrease in the parameter $N$.

Finally, the problem of angular momentum may be the most sorious multi-dimensional effect. The hot electrons from the channels will have largo non-radial velocity components, which 
will grobably keop then from reaching the target. If the fields are indepenitent of he sphetical coordinates" and $\theta$, then

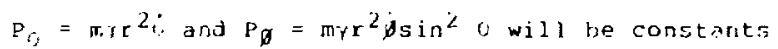
of the motiun. [f, eq.. an e ectron has $v_{u}=1 ;=1 \times 10^{10}$ cin'su (a conservatime value) at $r_{1}=4 \mathrm{~cm}$, thrir the minimis possinle radius for this elsctoon $i=1 \frac{1}{3} \mathrm{~cm}$, and this upes aot include the reduction in as the alectron passas through the potential well:

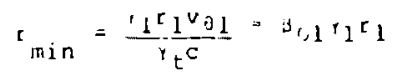

where subsceint "l" cienot v values ot $r_{1}$, while "t" means tulget. Unless one invokes the malic words "turbulent electris I iflds" or "anomalous diffusion," it 15 difficult to sce why Ey. $(10)$ dors not make the whole e - i convertes scheme of dubious viability. Even if non-radial E - fields are presant with allow enduth electrons to rach the tarat, thegr w:ll detirus the ions.

\section{v. Corclusions}

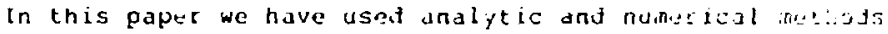
to study the e - i convertor. The l-D results indlcate that interestingly large ( $\sim M A$ ) ion curents nay reach the target, but the power efficiency of the process is anly $\cdot 108$. Folding in doleterious 2-D effects, especially angular momentum, the scheme may be expected to have serious difficulties. 


\section{Acknowledgment}

The author had a number of useful discussions about this problem with P. A. Miller, 4244. 
Reftrences

1. P. A. Miller, R. I. Butler, M. Cowan, J. R. Froman,

J. W. Poukey, T. P. Wright, and G. Yonas, whys. Rev.

Lett. 39, 92 (1977).

2. T. P. Wright, J. Appl. Phys. 49, 3842 (1978).

3. P. A. Miller and L. P. Mix, Bull. Am. Phys. Sวc. 23, $8: 3(1978)$.

4. P. A. Miller, Internal Memo, Sept. 1978.

5. S. A. Goldstein and I. Guillory, Phys. Rev. Lett. 35 , $1160(1975)$.

5. J. T. Verdeyen, D. A. Swanson, B. E. Cherrington, and W. L. Johnson, Appl. Phys. Lett. 27, 380 (1975).

7. K. L. Hirsch, Phys. Fluids 1I, 2486 (1968).

8. C. W. Barnes, Stanford University Institute for Plasma Research Report No. 344, March 1970.

4. J. W. Poukey, J. R. Freeman, M. J. Clauser, and G. Yonrs, Phys. Rev. Lett. 35, 1806 (1975).

10. I. Langmuir and K. B. Blodgett, Phys. Rev. 24, 49 (1924).

11. C. B. Wheeler, J. Phys. A. 10, 1645 (1977).

12. Y. X. Winograd, Phys. Fluids 10,1063 (1967).

13. C. K. Birdsall and W. B. Bridges, J. Appl. Phys 32, 2611 $(1961)$.

14. J. W. Poukey and N. Rostoket, Plas. Phys. 13, 897 (1971), (Fig. 4), and references therein.

15. P. A. Miller, private communication.

16. A Sternilew, Shyke A. Goldstein, and R. Lee, U. of Maryland Report 79-059, Dec. 1978. 